\title{
Optimal ambulatory vital sign targets in pulmonary hypertension pregnant women via time-dependent survival analysis
}

\author{
renhuai liu ${ }^{1}$, ziyu zheng ${ }^{1}$, and binxiao $\mathrm{su}^{1}$ \\ ${ }^{1}$ Air Force Medical University Xijing Hospital
}

January 26,2022

\begin{abstract}
Objective To investigate the safe range of vital signs of pregnant women pulmonary hypertension (PH). Study design Retrospective study. Setting The largest First-class Hospital at grade 3 in Northwestern China Population pregnant women with PH in Intensive care unit (ICU). Methods This of consecutive obstetric patients with PH admitted to ICU of the First Affiliated Hospital of Air Force Military Medical University of China, from January 2011 to May 2020, consisted of 92 cases analyzed using time-dependent Cox regression to consider the dynamic features of vital signs. Main outcome measures Maternal mortality Results 7/92 maternal deaths occurred. Three vital signs were identified as risk factors in the maternal in-hospital mortality model via backward selection: $\mathrm{SpO} 2(\mathrm{HR}, 0.93 ; 95 \% \mathrm{CI}, 0.88-0.97 ; \mathrm{P}=0.003)$, heart rate $(\mathrm{HR}, 0.94 ; 95 \% \mathrm{CI}, 0.90-0.99 ; \mathrm{P}=0.027)$, and mean arterial pressure (MAP) $(\mathrm{HR}, 1.09 ; 95 \% \mathrm{CI}, 1.00-1.18 ; \mathrm{P}=0.045)$. The optimal range of $\mathrm{SpO} 2<73 \%$, MAP was $65-95 \mathrm{mmHg}$, and heart rate was 59-125 beats per minute (bpm). Further exploration showed that the cumulative and the longest consecutive time of abnormal vital signs also affect the outcome. For example, SpO $2<73 \%$ accumulated for 5 h or continuously up to 2 $\mathrm{h}$ increases mortality. Conclusions: Maintaining SpO2>73\%, MAP at $65-95 \mathrm{mmHg}$, and heart rate at 59-125 bpm can significantly reduce in-hospital maternal mortality. The effects of the abnormal SpO2, heart rate, and MAP on in-hospital maternal mortality should be combined with the cumulative time and the longest duration. Funding Dr. Binxiao Su is supported by the National Institutes of Health (NIH) grant 81870961
\end{abstract}

\section{Introduction}

Pulmonary hypertension $(\mathrm{PH})$ is a progressive and devastating disease that often leads to right ventricular failure and death if untreated ${ }^{1}$. The significant fluctuations during pregnancy often aggravate the hemodynamic abnormalities for $\mathrm{PH}$ patients, especially those during deliveries or at early-postpartum periods. These phenomena further increase the burden of the heart and lead to maternal heart failures or even deaths ${ }^{2}$. Therefore, maternal mortality is high among PH patients $(17-33 \%)^{3}$. The mortality is elevated to around $50 \%$ for pregnant women with Eisenmenger syndrome, which severely affects the health and safety of pregnancy ${ }^{4}$. The European Society of Cardiology (ESC) guidelines strongly recommend that women with $\mathrm{PH}$ avoid pregnancy for health and safety reasons ${ }^{5}$. Some women continue with unplanned pregnancies or even choose to become pregnant despite such conditions. Pregnant women with PH must be referred to a $\mathrm{PH}$ center for multidisciplinary management for intensive postoperative monitoring. Being one of the most resourceful units in the hospital, the intensive care unit (ICU) facilitates continuous individual monitoring ${ }^{6,7}$.

Hypoxia, hypotension, and arrhythmias are commonly associated with PH patients ${ }^{8}$ and adverse outcomes ${ }^{8}$. Thus, the intensivists should focus on the management of vital signs within a reasonable range. However, an optimal target range of vital signs in critically ill patients with $\mathrm{PH}$ has not yet been defined, especially for pregnant patients with PH. Although the ESC guidelines recommend oxygen $\left(\mathrm{O}_{2}\right)$ administration when arterial $\mathrm{O}_{2}$ saturation is $<91 \%$, the $\mathrm{O}_{2}$ therapy is based on the evidence of patients with chronic obstructive pulmonary disease $(\mathrm{COPD})^{8}$. Owing to the special conditions and potentially high risks, in-depth insights into the management of vital signs are essential for pregnant women with $\mathrm{PH}$. 
Since the vital signs are the first step to preventing and managing critical illness ${ }^{11}$, they are crucial to the indication of the patients at risk of deterioration. These signs are also first-hand observations containing abundant information easily available in the ICU. Hence, tracking dynamic, vital signs to alert doctors to potential risk allows early actions to prevent potentially catastrophic maternal adverse outcomes.

Therefore, the present study provided a different approach to investigate the survival of pregnant women with PH: time-dependent Cox regression. A retrospective study on pregnant women with $\mathrm{PH}$ on admission to ICU was conducted to investigate the potential of ambulatory vital sign data. The optimal range of vital sign management was evaluated, and the correlation between the duration of abnormal vital signs and risk of death was calculated. Together, these findings provided an adequate reference to physicians for evaluating and managing specific patients.

\section{Methods}

\section{Study design and subjects}

This retrospective study consisted of consecutive obstetric patients with $\mathrm{PH}$ admitted to the ICU of the First Affiliated Hospital of Air Force Military Medical University (Xi'an, Shaanxi, China), from January 2011 to May 2020. All study subjects were either pregnant or within 42 days of postpartum, suffering from $\mathrm{PH}$ conditions. The data of 92 pregnant women with $\mathrm{PH}$ were extracted from the electronic medical record system (EMRS). Further verification of these study subjects was conducted using the registration books of the ICU department. The present study was approved by the ethics committee (KY20212019).

\section{Data collection and definition}

$\mathrm{PH}$ is defined as an increase in the mean pulmonary arterial pressure at rest (mPAP [?]25 $\mathrm{mmHg}$ ) assessed by the right heart catheterization $(\mathrm{RHC})^{11}$. Despite an established gold standard tool of diagnostics for PAH, the RHC is an invasive procedure. Thus, for this study, the PAP is also monitored via echocardiography for all study subjects with $\mathrm{PH}$ on admission to the ICU. All values at the peak velocity tricuspid regurgitation were considered for consistency in the diagnostic criteria when estimating the systolic pulmonary arterial pressure (sPAP). Although the 2015 ESC guidelines for PH do not recommend the SPAP as a diagnostic and stratification criterion, it is widely used in clinical practice due to its intuitive and simple features. Hence in this study, all patients with right ventricular systolic pressure (RVSP) $>30 \mathrm{mmHg}$ at rest show high $\mathrm{PH}^{14}$. Patients are further stratified into three groups based on RVSP: $30-50 \mathrm{mmHg}, 50-70 \mathrm{mmHg}$, and $>70 \mathrm{mmHg}^{15,16}$. These measurement values were obtained by echocardiography.

The following information was recorded in the registry: age, chief complaint, medical history, latest echocardiographic, childbearing history, mode of delivery, ante/postpartum admission, anesthetic mode, length of stay in the ICU and hospital, and conditions on discharge. The information during the operation included the name of the operation, diagnosis upon ICU admission, acute physiology and chronic health evaluation II (APACHE II) score. Vital signs are also continuously monitored in the ICU with appropriate management.

\section{Statistical analysis}

All statistical analyses were performed using SPSS 26 (IBM Corporation, Armonk, NY,

USA) and R software (R Foundation for Statistical Computing, Vienna, Austria, 2016). The time-to-event data were evaluated based on survival analysis where the response is the time between the entry of the study (admission to ICU) and the event (death or discharge from ICU). Thus, in the present study, the response variable is the total time spent in the ICU $(\mathrm{h})$, where the data are assumed to be right-censored with deaths marked as 1. The Kaplan-Meier method is used to estimate and visualize survival probabilities as a function of survival time for exploratory analysis.

$$
h_{i}(t)=\phi\left(x_{i}(t)\right) h_{0}(t)
$$


The advanced monitoring technologies collect the longitudinal data after ICU admission. Based on the assumption that the dynamic features of vital signs contribute to the final outcome (for instance, mortality in this paper), the inclusion of time-varying covariates is necessary. Herein, the dynamically monitored data were utilized in the Cox regression with time-dependent covariates to allow the inclusion of potential timevarying effects. Such a model takes into account the time-varying covariates $\mathrm{x}(\mathrm{t})$ that controls the hazard of mortality. is the baseline hazard function, which describes the legitimate failure rate when risk factors are at baseline values. The hazard for the individual in the study is as follows: . According to the time-varying effects, the model also automatically releases the assumption of proportionality and allows a flexible intake of factors. $P$-value $<0.05$ indicated statistical significance.

\section{Results}

\section{Baseline characteristics}

A total of 113 pregnant women diagnosed with $\mathrm{PH}$ were admitted to ICU within the last decade in the First Affiliated Hospital of Air Force Military Medical University of China, of which 7 were excluded for the absence of ultrasound examination or unqualified diagnostic criteria and 14 were excluded for lack of vital signs; finally, 92 patients were analyzed in this study (Figure 1). The mean maternal age of these cases was 27.05 (range: 17-42)-years-old. The mean gestational number of weeks of cases admitted to the ICU was $31.8 \pm 6.9$; among them, $63(68.5 \%)$ were nulliparous. The in-hospital maternal mortality was $7.6 \%$, and most of the admissions were due to postpartum or miscarriage. The cohort comprised 71 pregnancy cases that underwent cesarean section, 2 had a vaginal delivery, 15 received hysterotomy, and 4 had an abortion. Among them, 67 were live births, and 48 were premature. The sPAP of the cases was $83.4 \pm 34.3$, where about $55.5 \%$ suffered from severe $\mathrm{PH}(\mathrm{sPAP}>70 \mathrm{mmHg})$. The APACHE II score of the cases on admission to the ICU was $9.1 \pm 5.2$. The average stay in ICU was about 2.9 days with an outlier of 14 days; $75 \%$ of patients were discharged from the ICU in 3 days. The clinical characteristics of these cases are shown in Table 1.

\section{Trends of vital signs in the ICU}

The mean temporal trends in a time-dependent manner across the survival and death groups are shown in Figure 2. The vital signs for the survival group tend to be more stable and lie within the normal range compared to the non-survival group. Furthermore, the death group presented low $\mathrm{SpO}_{2}$ and high heart rate. Such observational differences were observed between two trends and fluctuations in durations, which necessitated the assessment of dynamic, vital signs in pregnant women with $\mathrm{PH}$.

\section{Vital signs associated with maternal death}

The dynamically monitored vital signs were analyzed via time-dependent Cox regression. All five relevant vital signs $\left(\mathrm{SpO}_{2}\right.$, heart rate, MAP, temperature, and respiration) were considered initially. The model selection was based on the backward selection until the most optimal model was established with the indication of Akaike information criterion (AIC) values (optimal model with $\mathrm{AIC}=15.1$ ). The final model identified $\mathrm{SpO}_{2}$ (hazard ratio (HR): 0.93; 95\% confidence interval (CI): 0.88-0.97), heart rate (HR: 0.94; 95\% CI: 0.90-0.99), and MAP (HR: 1.09; 95\% CI: 1.00-1.18) as risk factors in describing the instantaneous hazard of death for these patients (Table 2).

\section{Safe range of predictors based on adjusted HRs}

The adjusted relative hazard at log-scale for each risk factor is given in Figure 3. The solid lines and shaded areas present estimated log relative HRs with $95 \%$ CIs. The factors exert a protective effect if the log-scaled relative hazard is $<0$, otherwise risk effect. The risk range calculated for heart rate is $<59$ or $>125$. $\mathrm{SPO}_{2}<73$ increases the hazard of death, while a MAP range between 65 and 95 is considered safe.

The survival probability adjusted for $\mathrm{SPO}_{2}, \mathrm{MAP}$, and heart rate is shown in Figure 4. The overall survival probability declined by $10 \%$ at around $72 \mathrm{~h}$ post-admission. This phenomenon suggested that the majority of the in-hospital mortality occurred outside the 72-h interval. Most of the patients were discharged within 3 days. 


\section{Survival probability of durations of abnormal vital signs}

A single-point observation does not contribute to the final outcome, but the duration is critical. Typically, the longer the vital signs of patients remain outside the safe range, the higher the risks of hazards. This duration differs for each risk factor. This section presents the adjusted survival probabilities of total duration outside the safe ranges for MAP, $\mathrm{SPO}_{2}$, and heart rate, respectively. The cutoff points are the first quartiles of overall duration within the risk range for all individuals in the study for each factor. The survival probabilities divert at around day 3 of ICU admission. A clear difference in the survival probability was observed for different durations of each risk factor outside the safe zone. A distinct decline was observed in $\mathrm{SPO}_{2}$, where if the patient suffered hypoxia $(<73 \%)$ for $>5 \mathrm{~h}$, the survival probability decreased by 0.25 . The difference in survival is smaller for both heart rate and MAP. For example, the duration of risk zones for heart rate $(<59$ $\mathrm{bpm}$ or $>125 \mathrm{bpm}$ ) was $2 \mathrm{~h}$ with about 0.05 drop in survival probability on day 3 of ICU admission. The survival probability differs when the duration for MAP $<65 \mathrm{mmHg}$ reaches $1 \mathrm{~h}$ or when the duration for MPA $>95 \mathrm{mmHg}$ reaches $9 \mathrm{~h}$ (Figure 5 ).

Similar to the total duration, Figure 6 shows the longest duration of consecutive times outside the safe range for each risk factor. Clear differences were detected compared to the total duration. For example, the survival probability is reduced by $50 \%$ in patients with $\mathrm{SPO}_{2}$ continuously $<73 \%$ for $>2 \mathrm{~h}$. For heart rate, the survival probability drops to 0.75 when the duration of low heart rate reaches $2 \mathrm{~h}$. The high heart rate showed less difference in survival when the abnormality lasted for $>1 \mathrm{~h}$ (around 0.2 ). MAP $>95 \mathrm{mmHg}$ for $>4 \mathrm{~h}$ showed a similar pattern in survival, but $\mathrm{MAP}<65 \mathrm{mmHg}$ for $>1 \mathrm{~h}$ presented minimal difference.

\section{Discussion}

Herein, the association between ambulatory vital signs and the survival outcomes of pregnant women with PH admitted to the ICU is described. The benefit is not only limited to the ability to forecast the instantaneous hazard of an individual at a given time adjusted for relevant risk factors but also utilizes the available information in the ICU. On general acceptance, pregnant women should be considered differently from others. This study fills the gap in the research field of $\mathrm{PH}$ based on this specific group.

A total of 92 cases with a mortality rate of $7.6 \%$ were identified. However, the rate was higher than 5/151 $(3.3 \%)$ reported by the European Registry of pregnancy and Cardiac Disease (ROPAC) ${ }^{16}$. Strikingly, sPAP is associated with poor outcome ${ }^{17,18}$. The present study contains a larger proportion of cases with sPAP (55.5\%). During ICU hospitalization, pregnant women with PH experienced long-term abnormal fluctuations in vital signs in the death group.

Since recording vital signs in the ICU is a dynamic process, the use of static vital signs might not be sufficient to reveal the underlying effects ${ }^{19,20}$. Typically, the vital signs averaged across individuals showed clear instability with a wide range of fluctuation for the death group; the variables were not similar while modeling the hazard. The employment of the time-dependent hazard model facilitates the impact of dynamically received ambulatory vital signs. The general proportion is true across survival durations, where risk factors fluctuate constantly. Thus in the case of vital signs, all varying values need to be considered. This limits the potential bias caused by single-point observation. On the other hand, it allows an ad-hoc update of the predictions about the survival outcome based on the observed risk factors. The estimated overall survival probability suggested that the instantaneous survival is reduced by roughly $10 \%$ after $72 \mathrm{~h}$ post-admission.

A correlation between the ambulatory vitals and the survival outcome is established and quantified by the $\mathrm{HR}$ of each factor. The average overall $\mathrm{SpO}_{2}, \mathrm{MAP}$, and heart rate are determined for statistical significance for the most optimal model fit. $\mathrm{SPO}_{2}$ is a protective factor, and the effects of heart rate and MAP were not treated as linear contributions towards the outcomes as the values above or below specific thresholds are considered in hazard zones. The relative hazard varies with different values of risk factors showed a trend for U-curves with respect to the association of heart rate and mean arterial pressure with log HR, negative linear for the association of $\mathrm{SPO}_{2}$ with $\log \mathrm{HR}$. The safe intervals indicated for MAP, heart rate, and $\mathrm{SPO}_{2}$ were $65-95 \mathrm{mmHg}, 59-125 \mathrm{bpm}$, and $73-100 \%$, respectively. Such a formula can be further used to calculate the survival for this specific group of patients. For example, the instantaneous hazard of death at the specific 
hour of admission to ICU can be obtained.

Low arterial oxygen saturations were associated with adverse outcomes. A retrospective study showed that $\mathrm{SpO}_{2}<88 \%$ had significantly poor survival in patients with interstitial lung disease-associated pulmonary hypertension ${ }^{21}$. The current study demonstrated that $\mathrm{SpO}_{2}<73 \%$ was associated with increased mortality. The difference in threshold from previous studies may be due to the diversity in the study population and duration of follow-up. Many patients admitted to the ICU with severe pulmonary hypertension are accompanied by hypotension and inotropic and vasopressor agents used commonly to ameliorate hypotension ${ }^{22}$, but recent guidelines do not include a range for blood pressure maintenance. The current data filled this gap by showing that MAP between 65 and $95 \mathrm{mmHg}$ was associated with low in-hospital mortality. Supraventricular tachyarrhythmia frequently occurs in patients with $\mathrm{PH}$ and is associated with increased morbidity ${ }^{23}$. The REVEAL registry demonstrated that heart rate $>92 \mathrm{bpm}$ is associated with an increased risk of death at 1 year in patients with $\mathrm{PH}^{24}$, while another study indicated that heart rate $<82 \mathrm{bpm}$ significantly prolonged the survival ${ }^{25}$. This study showed that the correlation between heart rate and in-hospital mortality was U-shaped; heart rate $>125 \mathrm{bpm}$ increased the in-hospital maternal mortality, and the safe minimum heart rate was $59 \mathrm{bpm}$. Therefore, the use of vasoactive drugs to correct hypotension with a moderate increase in heart rate is acceptable as long as the heart rate does not exceed $125 \mathrm{bpm}$.

The abnormal values of the vital signs are the complementary sets of the safe zones. Despite that the actual values of vital signs affect the final outcomes, how long the patient suffers in the abnormal zone is intuitive. The present study explored the impact of the duration of each vital sign on the survival outcome within the abnormal value zone. We observed that the longer the total duration within the abnormal values, the worse the survival outcomes after $72 \mathrm{~h}$. This is the case for $\mathrm{SpO}_{2}$, wherein the survival probability drops by 0.15 when the total duration reaches $5 \mathrm{~h}$ (the first quartile of the total duration for all individuals), while the corresponding quartiles of duration for heart rate and MAP do not show a significant decline in survival probability.

The consecutive durations within abnormal value zones exhibit a high identification in survival probabilities for all three vital signs. For instance, the survival probability for those who suffer hypoxia for $>5 \mathrm{~h}$ is reduced after $72 \mathrm{~h}$ post-admission to the ICU. Thus clinically, when low $\mathrm{SPO}_{2}$ alert is shown, it is not critical if measures are administered and the value is restored to the safety threshold, but when accumulated hypoxia duration is $>5 \mathrm{~h}$, the situation can be considered fatal. If the heart rate is low for $>2 \mathrm{~h}$ continuously, the survival chance drops by 0.15 , but if it is $<2 \mathrm{~h}$, the patients are likely to survive. Strikingly, when the heart rate is $>125 \mathrm{bpm}$, this phenomenon is distinct in $1 \mathrm{~h}$. Thus, it is crucial to maintain the duration of vital signs with abnormal values at least below these time thresholds.

From a clinical perspective, this retrospective analysis not only identified an optimal threshold for the management of maternal vital signs in the ICU with PH but also estimated the cutoff point for the total duration and maximum duration of abnormal vital signs that significantly increased the rate of mortality, thereby providing a reference for clinicians in the management of these patients. Moreover, this method can calculate the instantaneous mortality during hospitalization with the duration of abnormal vital signs over time. The obstetrics of this population filled the gaps in perinatal management. Also, clinicians routinely use vital signs for decision-making in the $\mathrm{ICU}^{26}$. Future studies would need to consider the dynamic trends of each vital sign, which would guide us in the area of pregnancy with $\mathrm{PH}$ and other potential cases within ICU. The real-time evaluation of patient conditions is also achievable in the future with advanced monitoring equipment and recording systems.

\section{Study limitations}

Nevertheless, the present study has some limitations. A potential bias due to the small sample size for Cox regression modeling cannot be ignored. This might limit the total number of study subjects since $\mathrm{PH}$ is a rare disease in a pregnant population. However, the hospital from which the data is collected is the largest First-class Hospital at grade 3 in Northwestern China, wherein the population accounts for most of the targeted patients within the region and thus is sufficiently representative. The statistical model is robust, 
wherein the results need to be interpreted in combination with other background information. All the data sources are authentic and reliable as it was a single-center study.

\section{Conclusion}

The current results proposed a strategy to maintain the MAP between 65 and $95 \mathrm{mmHg}$, heart rate between 59 and $125 \mathrm{bpm}$, and $\mathrm{SpO}_{2}>73 \%$, which reduces the risk of in-hospital maternal mortality. The cumulative and the longest duration under abnormal conditions are determined for each vital sign. The cumulative time and the longest duration of $\mathrm{SpO} 2, \mathrm{MAP}$, and heart rate were associated with low in-hospital mortality under abnormal conditions. These findings need to be substantiated further in clinical trials to confirm the optimal reference target of vital signs of pregnant women with $\mathrm{PH}$.

Abbreviation: $\mathrm{AIC}=$ Akaike information criterion; $\mathrm{AUC}=$ area under the receiver operating characteristic curve; APACHE II=acute physiology and chronic health evaluation II; HR=hazard ratio; $\mathrm{ICU}=$ intensive care unit; $\mathrm{MAP}=$ mean arterial pressure; NYHA: New York Heart Association; OR=odds ratio; $\mathrm{PAP}=$ pulmonary arterial pressure; $\mathrm{PH}=$ pulmonary hypertension; $\mathrm{ROC}=$ receiver operating characteristic curve; REVEAL=Registry to Evaluate and Long-Term PAH disease management; $\mathrm{SD}=$ standard deviation; $\mathrm{sPAP}=$ pulmonary arterial systolic pressure; $\mathrm{COPD}=$ chronic obstructive pulmonary disease

\section{Declarations}

\section{Acknowledgements}

Not applicable.

\section{Authors' contributions}

Renhuai Liu and Ziyu Zheng drafted the manuscript, had full access to all the data, and takes responsibility for the integrity of the data and the accuracy of the data analysis. Binxiao Su contributed to study conception, design ,interpretation and critical revision.

\section{Availability of data and materials}

The datasets used and/or analyzed during the current study are available from the corresponding author on reasonable request.

\section{Ethics approval and consent to participate}

The study was approved by Ethic Committee of Xijing Hospital,Fourth Military Medical University (Approved NO. of ethic committee :KY20212019). As it was a retrospective analysis, consent to participate does not apply.

\section{Consent for publication}

Not applicable.

\section{Competing interests}

The authors declare that they have no competing interests.

\section{References:}

1 Farber HW, Loscalzo J. Pulmonary arterial hypertension. N Engl J Med 2004; 351:1655-1665

2 Ramlakhan KP, Johnson MR, Roos-Hesselink JW. Pregnancy and cardiovascular disease. Nat Rev Cardiol 2020; 17:718-731

3 Duarte AG, Thomas S, Safdar Z, et al. Management of pulmonary arterial hypertension during pregnancy: a retrospective, multicenter experience. Chest 2013; 143:1330-1336

4 Bedard E, Dimopoulos K, Gatzoulis MA. Has there been any progress made on pregnancy outcomes among women with pulmonary arterial hypertension? Eur Heart J 2009; 30:256-265 
5 N G, M H, JL V, et al. 2015 ESC/ERS Guidelines for the diagnosis and treatment of pulmonary hypertension: The Joint Task Force for the Diagnosis and Treatment of Pulmonary Hypertension of the European Society of Cardiology (ESC) and the European Respiratory Society (ERS): Endorsed by: Association for European Paediatric and Congenital Cardiology (AEPC), International Society for Heart and Lung Transplantation (ISHLT). Eur Heart J 2016; 37:67-119

6 SV K. Trends in pregnancy outcomes in patients with pulmonary hypertension: still a long way to go. Eur J Heart Fail 2016; 18:1129-1131

7 DG K, R C, V W, et al. Improved survival in pregnancy and pulmonary hypertension using a multiprofessional approach. BJOG : an international journal of obstetrics and gynaecology 2010; 117:565-574

8 Olsson KM, Channick R. Pregnancy in pulmonary arterial hypertension. Eur Respir Rev 2016; 25:431-437

$9 \mathrm{M} \mathrm{D}, \mathrm{H} \mathrm{N}, \mathrm{A} \mathrm{K}$, et al. Arrhythmias in adult patients with congenital heart disease and pulmonary arterial hypertension. Heart (British Cardiac Society) 2018; 104:1963-1969

10 Weitzenblum E, Sautegeau A, Ehrhart M, et al. Long-term oxygen therapy can reverse the progression of pulmonary hypertension in patients with chronic obstructive pulmonary disease. Am Rev Respir Dis 1985; 131:493-498

11 Cummings MJ, Wamala JF, Bakamutumaho B, et al. Vital signs: the first step in prevention and management of critical illness in resource-limited settings. Intens Care Med 2016; 42:1519-1520

12 MM H, HJ B, R C, et al. Definitions and diagnosis of pulmonary hypertension. J Am Coll Cardiol 2013; 62:D42-D50

13 G S, D M, DS C, et al. Haemodynamic definitions and updated clinical classification of pulmonary hypertension. The European respiratory journal 2019; 53

14 Strange G, Stewart S, Celermajer DS, et al. Threshold of Pulmonary Hypertension Associated With Increased Mortality. J Am Coll Cardiol 2019; 73:2660-2672

15 Martin SR, Edwards A. Pulmonary Hypertension and Pregnancy. Obstet Gynecol 2019; 134:974-987

16 Sliwa K, van Hagen IM, Budts W, et al. Pulmonary hypertension and pregnancy outcomes: data from the Registry Of Pregnancy and Cardiac Disease (ROPAC) of the European Society of Cardiology. Eur J Heart Fail 2016; 18:1119-1128

17 MM H, T K, Z P, et al. Mortality in pulmonary arterial hypertension: prediction by the 2015 European pulmonary hypertension guidelines risk stratification model. The European respiratory journal 2017; 50

18 Frea S, Pidello S, Bovolo V, et al. Prognostic incremental role of right ventricular function in acute decompensation of advanced chronic heart failure. Eur J Heart Fail 2016; 18:564-572

$19 \mathrm{CD}$ J, RH O, LR P, et al. Resting, night-time, and $24 \mathrm{~h}$ heart rate as markers of cardiovascular risk in middle-aged and elderly men and women with no apparent heart disease. Eur Heart J 2013; 34:1732-1739

20 G P, G S, E O, et al. European Society of Hypertension practice guidelines for ambulatory blood pressure monitoring. J Hypertens 2014; 32:1359-1366

21 Alhamad EH, Cal JG, Alrajhi NN, et al. Predictors of Mortality in Patients with Interstitial Lung Disease-Associated Pulmonary Hypertension. J Clin Med 2020; 9

22 Aryal S, King CS. Critical care of patients with pulmonary arterial hypertension. Curr Opin Pulm Med 2020; 26:414-421

23 Tongers J, Schwerdtfeger B, Klein G, et al. Incidence and clinical relevance of supraventricular tachyarrhythmias in pulmonary hypertension. Am Heart J 2007; 153:127-132 
24 Benza RL, Miller DP, Gomberg-Maitland M, et al. Predicting survival in pulmonary arterial hypertension: insights from the Registry to Evaluate Early and Long-Term Pulmonary Arterial Hypertension Disease Management (REVEAL). Circulation 2010; 122:164-172

25 Hildenbrand FF, Fauchere I, Huber LC, et al. A low resting heart rate at diagnosis predicts favourable long-term outcome in pulmonary arterial and chronic thromboembolic pulmonary hypertension. A prospective observational study. Respir Res 2012; 13:76

26 BD W, SJ W, ER P, et al. Rapid-response systems as a patient safety strategy: a systematic review. Ann Intern Med 2013; 158:417-425

\section{Hosted file}

image4.emf available at https://authorea.com/users/457381/articles/554187-optimal-ambulatoryvital-sign-targets-in-pulmonary-hypertension-pregnant-women-via-time-dependent-survivalanalysis

Figure 1. Flowchart of the study.

Table 1 Clinical characteristics of the study population

Patients $(\mathrm{N}=92)$

Baseline characteristics Age, y (SD) $27.05 \pm 5.7$ (17-42) Nulliparous 63 (68.5\%) Gravidity $2.1 \pm 1.2$ Gestational week $31.8 \pm 6$. NYHA class I 53 (57.6\%) II $11(12.0 \%)$ III 16 (17.4\%) IV $12(13.0 \%)$

Right ventricular systolic pressure $(\mathrm{mmHg})$

$30-5019(20.7 \%)$

$50-7022(23.9 \%)$

70-90 $10(10.9 \%)$

$>9041(44.6 \%)$

Mode of delivery Vaginal 2 (2.2\%) Cesarean section 71 (77.2\%) Hysterotomy 15 (16.3\%) Abortion 4 (3.5\%)

Fetal outcome Live birth 67 (72.8\%) Premature 48 (52.2\%)

Length of ICU stay, days 2.9 \pm 2.0 (1-14) Maternal death, N (\%) 7 (7.6\%)

NYHA: New York Heart Association

Table 2. Multivariable Cox regression analysis for the maternal in-hospital death.

\begin{tabular}{llll}
\hline Predictors & Hazard ratio & $95 \%$ Confidence interval & $P$-value \\
\hline $\mathrm{SpO}_{2}$ & 0.93 & $0.88-0.97$ & 0.003 \\
Heart rate & 0.94 & $0.90-0.99$ & 0.027 \\
Mean arterial pressure & 1.09 & $1.00-1.18$ & 0.045 \\
\hline
\end{tabular}

\section{Hosted file}

Figure.doc available at https : //authorea.com/users/457381/articles/554187-optimal-ambulatoryvital-sign-targets-in-pulmonary-hypertension-pregnant-women-via-time-dependent-survivalanalysis 